Review

\title{
Utility of Fungal Cell Markers in Pediatric Bone Marrow Transplant Recipients for Screening and Diagnosis of Invasive Fungal Infections
}

Nour Hasan ${ }^{1}$, Ankita P. Desai ${ }^{1,2}$, Jignesh Dalal ${ }^{1,2, *}$

1. Rainbow Babies and Children's Hospital, Cleveland, OH, USA; E-Mails:

nour.hasan@uhhospitals.org; Ankita.Desai@UHhospitals.org; Jignesh.Dalal@UHhospitals.org

2. Case Western Reserve School of Medicine, Cleveland, OH, USA

* Correspondence: Jignesh Dalal; E-Mail: Jignesh.Dalal@UHhospitals.org

Academic Editor: Dora Ho

Special Issue: Infectious Complications in Hematopoietic Stem Cell Transplantation

OBM Transplantation

2020, volume 4, issue 1

doi:10.21926/obm.transplant.2001098
Received: November 12, 2019

Accepted: January 22, 2020

Published: February 03, 2020

\begin{abstract}
Invasive fungal disease (IFD) is a significant contributor to morbidity and mortality in pediatric patients undergoing hematopoietic stem cell transplantation (HSCT). Early detection and timely initiation of antifungal therapy is important for improved outcome, but diagnosis remains difficult, especially when relying on conventional microbiology methods such as culture and microscopy. Data on the use of fungal biomarkers for screening and diagnosis of IFD in HSCT pediatric patients are limited, precluding the development of specific guidelines in this population. In this review, we have summarized available literature on kinetics, diagnostic and prognostic performance, and limitations of fungal biomarkers for screening and diagnosis of IFD in pediatric HSCT recipients to help guide their use and interpretation.
\end{abstract}

\section{Keywords}

$\beta$-D-glucan; galactomannan; invasive fungal disease; pediatric bone marrow transplant recipients

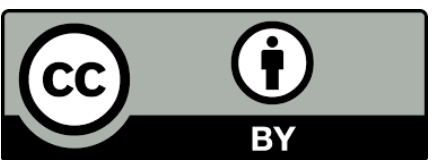

(C) 2020 by the author. This is an open access article distributed under the conditions of the Creative Commons by Attribution License, which permits unrestricted use, distribution, and reproduction in any medium or format, provided the original work is correctly cited. 


\section{Introduction}

Invasive fungal disease (IFD) is a significant contributor to morbidity and mortality in pediatric patients undergoing hematopoietic stem cell transplantation (HSCT). Early detection and timely initiation of antifungal therapy is important for improved outcome, but diagnosis remains difficult, especially when relying on conventional microbiology methods such as microscopy and culture. Use of biomarkers for early diagnosis of IFD in high risk patients have been introduced over the last 2 decades [1]. Galactomannan (GM) and 1,3- $\beta$-D-glucan are used as adjunctive diagnostic tools in adults and are now incorporated into the diagnostic criteria of IFD established by the Invasive Fungal Infections Cooperative Group of the European Organization for Research and Treatment of Cancer (EORTC) and the Mycoses Study Group (MSG) [2]. However, less data are available for children as compared to adults and pediatric guidelines are lacking specifically pertaining to their use for surveillance in asymptomatic pediatric HSCT recipients and their use in monitoring response to therapy. In this review, we have summarized available literature on kinetics, diagnostic and prognostic performance, and limitations of fungal biomarkers for screening and diagnosis of IFD in pediatric HSCT recipients to help guide their use and interpretation.

\section{1,3-ß-D-Glucan (BDG)}

\subsection{BDG}

BDG is the most important and abundant fungal wall polysaccharide of most fungi, estimated to comprise as much as $65-90 \%$ of total wall glucans[3]. BDG plays a role in the immune response to fungal infections through interaction with the macrophage through beta-glucan receptors[4]. An elevated serum BDG level has been shown to be a surrogate to invasive fungal infection. However, certain fungal infections may not result in detectable serum BDG levels including Cryptoccocus neoformans (encapsulation of the yeast), the yeast phase of Blastomyces dermatitidis, and zygomycetes [5-7]. With Blastomyces dermatitidis, it has been demonstrated that transition from the mould to the yeast form causes significant depletion of the cell wall BDG concentration [7]. The cell wall of zygomycetes consists of primarily chitin and chitosan. However, evidence suggests that the cell wall of zygomycetes may contain small amounts of $1,3-\beta-D$-glucan which might explain the reported susceptibility of $R$. oryzae to ecchinocandins, which inhibit the 1,3- $\beta$-D-glucan synthase as their mechanism of antifungal activity [8].

\subsection{BDG Detection Assays}

At least five commercial BDG detection assays have been developed [9, 10], but only the Fungitell assay is cleared by the Food and Drug Administration (FDA) in the United States. This assay is based on modification of the Limulus Amebocyte Lysate (LAL) pathway by eliminating reactivity to bacterial endotoxin so that it only reacts to 1,3- $\beta$-D-glucan, through the Factor Gmediated side of the pathway. When activated by BDG, factor $\mathrm{G}$ converts the inactive pro-clotting enzyme to the active clotting enzyme, which in turn cleaves a chromogenic substance, creating a 
chromophore, para-nitroaniline, which absorbs at $405 \mathrm{~nm}$. BDG concentration is then estimated based on the rate of optical density increase produced by the sample [10].

\subsection{Conditions Associated with Elevated BDG Other Than Fungal Infections}

It has been demonstrated that immunoglobulin products may have high burdens of BDG which may cause elevation in serum BDG with intravenous administration. BDG level may remain elevated for 3-4 days after discontinuation of immunoglobulin therapy but overall the duration and extent of that elevation depends on the brands, lots, and doses of immunoglobulin products administered [11]. Other blood products including albumin and blood coagulation factors may cause an elevation in serum BDG attributed to the cellulose membranes of depth-type filters used to process the blood $[12,13]$. Similarly, serum BDG level increases significantly after hemodialysis using cellulose membrane, which was not seen with hemodialysis using Cellulose triacetate, polymethyl methacrylate, or synthetic polysulfone membranes $[14,15]$. Elevation in BDG was also reported with a cupro-ammonium rayon membrane used in hemodialysis [16]. Additionally, some gauze products contain BDG to which transient elevation in serum BDG is attributed in postsurgical patients $[17,18]$. Some studies showed false-positive results (i.e., positive findings not related to invasive fungal infection) in patients with Pseudomonas bacteremia and Streptococcus pneumoniae bacteremia, which were not related to known causes of reactivity including use of fungal-derived antimicrobials [19]. Mennink-Kersten et al. determined the reactivity of BDG in several bacterial culture supernatants using the Fungitell assay. Among the clinical isolates tested, Alcaligenes faecalis, S. pneumoniae and Pseudomonas aeruginosa showed 1,3- $\beta$-D-glucan reactivity [19]. Marty et al. tested forty-four intravenous antimicrobial agents commercially available in the US for the presence of BDG using the Fungitell assay [20]. None of the tested antimicrobial solutions had detectable BDG at the usual drug maximum plasma concentration including piperacillin-tazobactam. In contrast, Liss et al. [21] reported detectable BDG levels in twenty-five ready-to-use antimicrobials including antifungals that may be enough to trigger a positive test in the serum.

\subsection{BDG Kinetics}

The metabolism pathway of $1,3-\beta$-D-glucan is not yet known [15]. $\beta$-glucanase does not exist in humans which precludes enzymatic degradation [11]. Ikemura et al. [11] reported that BDG was detected in the urine for only $2 \mathrm{~h}$ after administration (through BDG containing immunoglobulin products) in adult patients and that no more than $5 \%$ of the total amount administered was excreted in urine. Kato et al. estimated the median half-life of BDG in the plasma to be 20 hours (range 3.1 to 181.3 hours). There was no relationships between the plasma half-life of BDG and serum creatinine, creatinine clearance, urine volume, prothrombin activity, or serum albumin concentration which indicates that hepatic and renal functions have no effect on the half-life of plasma BDG [15]. Data specific to pediatric patients is not available. 


\subsection{Performance of Serial BDG to Screen for Invasive Fungal Infections in Pediatric Bone Marrow Transplant Recipients}

Data from a recent meta-analysis in adults suggests that BDG is a useful screening tool for invasive fungal diseases in high risk populations [22]. The pooled studies included patients with hematological malignancy, solid tumors, liver transplant, and other hospitalized patients who are at high risk for IFD. Patients with proven or probable IFDs who were diagnosed according to the EORTC/MSG classification [2], as well as patients with possible IFDs were also included in the analysis. The pooled sensitivity, specificity, positive likelihood ratio, negative likelihood ratio, and diagnostic odds ratio with $95 \%$ confidence intervals, were $0.82(0.68,0.90), 0.86(0.81,0.92), 5.85$ $(3.46,9.35), 0.22(0.12,0.39)$ and $26.52(11.72,60.07)$, respectively, using the Fungitell assay [22].

Only one prospective study published to date has specifically evaluated the value of serial BDG screening for the early detection of invasive fungal infections in pediatric patients undergoing allogeneic HSCT [23]. The results of this study showed that BDG screening has a relatively good sensitivity and a good negative predictive value, whereas the positive predictive value was less than 30\% (reported prevalence of invasive fungal disease was 17.6\%) suggesting limited usefulness of BDG application as a screening tool in HSCT patients. This study showed that the diagnostic performance of two consecutive positive BDG values was higher than a single one. Performance also varied based on the cut-off used and the time point at which the test was performed with relation to onset of fungal infection. When BDG was measured at the time of a first pathological result suggestive or consistent of IFD, such as blood culture results or biopsy sample, pulmonary infiltrates, or pathological GM test result, having a single BDG level higher than the respective cutoff value was associated with a sensitivity of $100 \%$ and specificity of $73 \%$ at a cutoff of $60 \mathrm{pg} / \mathrm{ml}$, and a sensitivity of $100 \%$ and specificity of $78 \%$ at a cut off of $80 \mathrm{pg} / \mathrm{ml}$. Having two consecutive positive BDG values above the cutoff used was associated with improved specificity to $78 \%$ and $82 \%$ at cut-off of $60 \mathrm{pg} / \mathrm{ml}$ and $80 \mathrm{pg} / \mathrm{ml}$, respectively. BDG had a lower sensitivity at 2 and 4 weeks prior to the first pathological signs. Up to 2 weeks prior to first pathological sign, two consecutive BDG values above the cutoff of $60 \mathrm{pg} / \mathrm{ml}$ had a sensitivity of $82 \%$ and specificity of $79 \%$. For two consecutive BDG values above the cutoff of $80 \mathrm{pg} / \mathrm{ml}$, the sensitivity was $69 \%$ with specificity of $83 \%$. At 4 weeks prior to first pathological sign, sensitivity was $70 \%$ and $58 \%$ at cutoff of $60 \mathrm{pg} / \mathrm{ml}$ and $80 \mathrm{pg} / \mathrm{ml}$, respectively. Associated specificities were $79 \%$ and $83 \%$ at cutoff of $60 \mathrm{pg} / \mathrm{ml}$ and 80 $\mathrm{pg} / \mathrm{ml}$, respectively [23].

\subsection{Diagnostic Performance of BDG in Pediatric HSCT Recipients with Suspected Fungal Infections}

Adult data suggests that BDG could be an adjunctive diagnostic tool for IFD in patients with appropriate pretest probability and symptoms suggestive of fungal infection, especially when checked serially in patients not receiving factors associated with falsely elevated BDG levels [24, 25].

Few studies evaluated BDG testing in hematology/oncology patients for diagnosis of IFD in pediatric patients whose clinical, radiological, or mycological signs were suspicious for a fungal infection [26, 27]. Badiee et al. [28] included patients with hematologic malignancies and did not include any HSCT recipients. This study reported poor diagnostic performance of BDG for diagnosis of invasive aspergillosis with reported sensitivity of $50 \%$, specificity of $46 \%$, positive likelihood 
ratio of 0.9 , and negative likelihood ratio of 0.9 . The prevalence of proven and probable aspergillosis in this cohort was $19.1 \%$ with a corresponding positive predictive value of $26 \%$ and a negative predictive value of $70.6 \%$. Guitard et al. [29] obtained serial measurements of BDG in patients with hematologic malignancies (the cohort did not include any HSCT recipient patients) during their febrile neutropenia episodes. The reported incidence of proven or probable fungal infections was $10.5 \%$. The sensitivity and specificity of BDG for detecting proven or probable IFD was $75 \%$ (95\% Cl, 69\%-81\%) and $56 \%$ (95\% Cl, 50\%-62\%), respectively using a BDG cut-off of 80 $\mathrm{pg} / \mathrm{ml}$. Using a lower cutoff was associated with higher sensitivity (75\%) and lower specificity. Having two consecutive positive samples increased the specificity to above $93 \%$, while decreased the sensitivity to $42 \%$. Performance of BDG was higher in patients not receiving antifungals prior to the test, approaching a sensitivity and specificity of $100 \%$ and $91 \%$, respectively, using a single positive BDG at a cut off of $75 \mathrm{pg} / \mathrm{ml}$. It is important to note that the majority of cases of proven or probable IFD in this cohort were secondary to yeast infections, and only one patient had Fusarium infection. Comparable results were reported by Gupta et al. [30] who included patients with hematologic malignancies and solid tumors. The following values were reported: sensitivity $80.2 \%$, specificity $47.2 \%$, PPV 68.9 and NPV $71.4 \%$. The reported incidence of invasive fungal infections was exceptionally high in that study, the author reported 2 proven and 60 probable cases of IFD out of the included 125 patients which would calculate to a prevalence of $49 \%$ of the cohort and did not report on the pathogens isolated. Zhao et al. [31] included pediatric patients with hematologic malignancies and HSCT recipients. In this study, the GKT-5M Set Kinetic Fungus Detection Kit was used for BDG assay. The sensitivity, specificity, positive and negative predictive values for plasma BDG detection were $81.8 \%, 82.4 \%, 48.6 \%$ and $95.7 \%$, respectively ( $16.9 \%$ prevalence of proven/probable IFD).

\subsection{BDG as a Prognostic Marker of Response to Treatment in Invasive Fungal Infections}

A decrease in BDG levels was reported to be associated with treatment response [32]. However, even with appropriate antifungal therapy, serum BDG declines slowly. Neither its height at diagnosis nor its early trajectory predicts clinical outcome or mortality [33]. It often lingers above the usual threshold for positivity long after clinical resolution of the original infection, reportedly for weeks to months, and up to 1.8 years after successful treatment [33,34]. Accordingly, negative BDG levels may not be a suitable marker for successful treatment outcome and has no discernible prognostic value after initiation of antifungal therapy.

\section{Galactomannan (GM)}

\subsection{Galactomannan and Conditions Associated with False Positive GM Reactivity}

Galactomannan is a polysaccharide component of the cell wall of Aspergillus species. It consists of a mannose backbone and a variable number of galactofuran side chains. GM antigens are secreted during mycelial growth $[35,36]$. The most commonly used method to determine GM in serum is a double sandwich enzyme-linked immunosorbent assay (ELISA) using EB-A2 monoclonal antibodies (Platelia Aspergillus antigen, Bio-Rad, Marnes-la-Cocquette, France) [37]. These antibodies bind to the $\beta$-(1,5)-galactofuranose (Galf) oligomers in the side chains of the galactomannan molecule. Other moulds including Penicillium and Paecilomyces also release 
antigens containing that epitope. The fungus Penicillium is used in the production process of certain $\beta$-lactam antibiotics, mainly piperacillin/tazobactam and amoxicillin/clavulanic acid, which explains the false positive reactivity of $\mathrm{GM}$ assays in the serum of patients receiving these antibiotics [38]. However, due to improvement of the manufacturing process, some currently available brand piperacillin/tazobactam preparations manufactured in the US no longer seem responsible for false-positive GM results [39, 40]. Galactoxylomannan released by Cryptococcus neoformans also cross-reacts with EB-A2 antibodies [38]. ELISA reactivity was also shown to be associated with bacterial lipoglycans containing a beta-1,5-galactofuranosyl chain in certain bacteria, most notably bifidobacteria. Since bifidobacteria are abundant in the human gut, especially in infants and neonates [41], these bacteria or the excreted lipoglycan may cause false serum GM reactivity [42]. False positive reactivity has also been reported with gluconatecontaining intravenous solutions [43] and infant milk formulas thickened with carob bean gum [44].

\subsection{GM Kinetics and Elimination}

Elimination of serum GM occurs via three different routes that include uptake by the kupffer cells in the liver, excretion in urine, and finally uptake by the neutrophils. The latter would explain the higher sensitivity of serum GM detection in neutropenic patients compared to nonneutropenic patients [36]. Aubry et al. [45] analyzed the kinetics of the decrease of GM in adult and pediatric patients with hematologic malignancies and bone marrow transplant recipients in whom an invasive aspergillosis was ruled out and source of GM reactivity deemed to be secondary to GM containing beta-lactam antibiotics. After discontinuation of implicated antibiotics, the average time to negative antigen was 5.5 days $(95 \% \mathrm{Cl}, 4.1$ to 7.0$)$, with an estimated half-life of elimination of $\mathrm{GM}$ of 2.4 days ( $95 \% \mathrm{Cl}, 1.8$ to 3.0 ).

\subsection{Performance of GM as a Screening and a Diagnostic Tool in Pediatric Bone Marrow Transplant Recipients for Detection of Invasive Fungal Infections}

A recent systematic review [46] of studies enrolling 184 patients with proven/probable IFD evaluated serum GM in the pediatric cancer and HSCT setting when used as a screening tool during immunosuppression or as a diagnostic test in patients presenting with symptoms potentially suggestive of an invasive fungal infection. Overall, the prevalence of IFD ranged from $0 \%$ to $30.8 \%$ in individual studies. Among the 10 studies in which GM was used for screening in patients with neutropenia or post-HSCT, wide ranges were observed as follows: sensitivity $0 \%$ 100\%, specificity 50\%-100\%, PPV 0\%-100\%, and NPV $85 \%-100 \%$. According to the authors, only 5 studies were appropriate for pooled analysis (used EORTC/MSG criteria, used proven or probable IFD as a case, and used a GM index $\geq 0.5$ once or twice, or $\geq 0.7$ once as a threshold for positive test). The pooled sensitivity and specificity for $\mathrm{GM}$ as a screening tool were $68 \%(95 \% \mathrm{Cl}, 51 \%-81 \%)$ and $91 \%(95 \% \mathrm{Cl}, 86 \%-94 \%)$ which calculates a positive likelihood ratio (LR) of 7.56 (95\% Cl, 3.6413) and a negative $\mathrm{LR}$ of $0.35(95 \% \mathrm{Cl}, 0.2-0.57)$ [46]. Eight studies including a total of 84 patients evaluated $\mathrm{GM}$ as a diagnostic test with the following reported ranges: sensitivity $14 \%-100 \%$, specificity $35 \%-100 \%$, PPV $0 \%-100 \%$, and NPV $70 \%-100 \%$. The pooled sensitivity and specificity of five studies were $89 \%(95 \% \mathrm{Cl}, 79 \%-95 \%)$ and $85 \%(95 \% \mathrm{Cl}, 51 \%-97 \%)$, respectively, which calculates a positive likelihood ratio (LR) of $5.93(95 \% \mathrm{Cl}, 1.61-32)$ and a negative LR of $0.13(95 \%$ 
$\mathrm{Cl}, 0.05-0.41$ ) [46]. Combining the results of both settings (diagnosis and screening) demonstrated a pooled sensitivity of $81 \%(95 \% \mathrm{Cl}, 69 \%-89 \%)$, pooled specificity of $88 \%$ ( $95 \% \mathrm{Cl}, 75 \%-95 \%)$, pooled positive likelihood ratio $6.75(95 \% \mathrm{Cl}, 2.76-18)$ and pooled negative likelihood ratio 0.22 (95\% Cl, 0.12- 0.41) [46]. For a pretest probability of $7 \%$ for invasive aspergillosis, a positive GM will raise the posttest probability to $36 \%$ (which may be enough of an increase to prompt additional diagnostic tests or initiation of antifungal therapy) and a negative GM will decrease the posttest probability to $1 \%$.

\subsection{GM as a Prognostic Marker of Response to Treatment in Invasive Fungal Disease}

It has been demonstrated in animal models that GM serum level was significantly associated with the degree of fungal burden of Aspergillus species in the lung. Receipt of appropriate antifungal therapy was associated with a decrease in GM levels and an increase in the survival rate [47]. Persistently positive serum GM despite antifungal therapy has been shown to correlate with persisting fungal burden and continuous fungal infection. Changes in GM from the time of diagnosis appear to significantly correlate with outcome [48], specifically, reduction in serum GM in the early phase of antifungal therapy, especially during the first one or two weeks, was reported to be associated with improved prognosis [48-51]. One study enrolling 45 pediatric patients with hematologic/oncologic diseases who were diagnosed with proven or probable invasive aspergillosis found that a serum $\mathrm{GM}>1.50$ at 1 week after initiation of therapy exhibited a sensitivity and specificity of $61.5 \%$ and $89.3 \%$, respectively, in predicting mortality within 12 weeks after antifungal therapy [47]. Another study that enrolled adult and pediatric patients with proven or probable invasive aspergillosis, including allogeneic bone marrow transplant recipients, found that each GM EIA unit decline in the week following diagnosis decreased the risk of time to allcause mortality at 6 weeks by 22\% [49]. Similarly, an increase in the GM value of 1.0 above the baseline value during the first week of treatment was predictive of treatment failure with a sensitivity of $44 \%$, a specificity of $87 \%$, and a positive predictive value of $94 \%$ in allogeneic HSCT recipients during treatment and suggests need for modification of treatment [50]. A systematic review of studies including adult and pediatric patients with hematologic malignancies including those undergoing allogeneic HSCT reported a very strong correlation between GM (measured one week before outcome) and survival with a k correlation coefficient of $0.8737(95 \% \mathrm{Cl}, 0.8140-$ 0.9333; $P<0.001$ ). Comparable results were demonstrated among the subgroups of studies evaluating adults, children, or a combination of both and among studies that included allogeneic HSCT recipients [51].

\section{Conclusions}

Given limited evidence, strong recommendations for routine use of BDG and GM in HSCT cannot be made. For surveillance in the early post HSCT period, BDG and GM seem to have a PPV less than $30 \%$ and $50 \%$, respectively, assuming a prevalence of $10 \%$ of IFD. While they may help identify invasive fungal infections in less than a half of the patients with a positive value, they may lead to unnecessary extensive workup in the other majority. Data for the use of BDG in pediatric HSCT recipients who are already exhibiting symptoms suggestive of IFD is lacking. Only a very small number of HSCT patients was included in the already published studies as discussed above and specific recommendations for its use cannot be made based on the limited available evidence. 
This population of patients may be different from other patients with hematologic malignancies in regards to the risk of IFD, receipt of prophylactic antifungal agents, and presence of factors associated with positive BDG in the absence of IFD, such as IVIG. All of these factors are significant determinants of the diagnostic performance of BDG as previously discussed. Overall, fungal cell markers, if used in symptomatic patients, should be interpreted in combination with the clinical and radiologic findings. Further research is needed to determine the value of using these markers in this specific group of patients.

\section{Author Contributions}

These authors contributed equally to this work.

\section{Competing Interests}

The authors have declared that no competing interests exist.

\section{References}

1. Lehrnbecher T, Hassler A, Groll AH, Bochennek K. Diagnostic approaches for invasive aspergillosis-specific considerations in the pediatric population. Front Microbiol. 2018; 9: 518.

2. De Pauw B, Walsh TJ, Donnelly JP, Stevens DA, Edwards JE, Calandra T, et al. Revised definitions of invasive fungal disease from the european organization for research and treatment of cancer/Invasive fungal infections cooperative group and the national institute of allergy and infectious diseases mycoses study group (EORTC/MSG) consensus group. Clin Infect Dis. 2008; 46: 1813-1821.

3. Ruiz-Herrera J, Ortiz-Castellanos L. Cell wall glucans of fungi. A review. The Cell Surface. 2019; 100022.

4. Miyazaki T, Kohno S, Mitsutake K, Maesaki S, Tanaka K, Hara K. (1-->3)-beta-D-glucan in culture fluid of fungi activates factor $G$, a limulus coagulation factor. J Clin Lab Anal. 1995; 9: 334-339.

5. Miyazaki T, Kohno S, Mitsutake K, Maesaki S, Tanaka K, Ishikawa N, et al. Plasma (1-->3)-betaD-glucan and fungal antigenemia in patients with candidemia, aspergillosis, and cryptococcosis. J Clin Microbiol. 1995; 33: 3115-3118.

6. Odabasi Z, Paetznick VL, Rodriguez JR, Chen E, McGinnis MR, Ostrosky-Zeichner L. Differences in beta-glucan levels in culture supernatants of a variety of fungi. Med Mycol. 2006; 44: 267272.

7. Girouard G, Lachance C, Pelletier R. Observations on (1-3)-beta-D-glucan detection as a diagnostic tool in endemic mycosis caused by Histoplasma or Blastomyces. J Med Microbiol. 2007; 56: 1001-1002.

8. Battaglia E, Benoit I, van den Brink J, Wiebenga A, Coutinho PM, Henrissat B, et al. Carbohydrate-active enzymes from the zygomycete fungus rhizopus oryzae: A highly specialized approach to carbohydrate degradation depicted at genome level. BMC Genomics. 2011; 12: 38.

9. Theel ES, Doern CD. B-D-glucan testing is important for diagnosis of invasive fungal infections. J Clin Microbiol. 2013; 51: 3478-3483. 
10. Wright WF, Overman SB, Ribes JA. (1-3)- $\beta$-D-glucan assay: A review of its laboratory and clinical application. Lab Med. 2011; 42: 679-685.

11. Ikemura K, Ikegami K, Shimazu T, Yoshioka T, Sugimoto T. False-positive result in limulus test caused by Limulus amebocyte lysate-reactive material in immunoglobulin products. J Clin Microbiol. 1989; 27: 1965-1968.

12. Usami M, Ohata A, Horiuchi T, Nagasawa K, Wakabayashi T, Tanaka S. Positive (1-->3)-beta-Dglucan in blood components and release of (1-->3)-beta-D-glucan from depth-type membrane filters for blood processing. Transfusion. 2002; 42: 1189-1195.

13. Liss B, Cornely OA, Hoffmann D, Dimitriou V, Wisplinghoff H. 1,3-ß-D-glucan concentrations in blood products predict false positive post-transfusion results. Mycoses. 2016; 59: 39-42.

14. Kanda H, Kubo K, Hamasaki K, Kanda Y, Nakao A, Kitamura T, et al. Influence of various hemodialysis membranes on the plasma (1-->3)-beta-D-glucan level. Kidney Int. 2001; 60: 319-323.

15. Kato A, Takita T, Furuhashi M, Takahashi T, Maruyama Y, Hishida A. Elevation of blood (1-->3)beta-D-glucan concentrations in hemodialysis patients. Nephron. 2001; 89: 15-19.

16. Yoshioka T, Ikegami K, Ikemura K, Shiono S, Uenishi $M$, Sugimoto $H$, et al. A study on limulus amebocyte lysate (LAL) reactive material derived from dialyzers. Jpn J Surg. 1989; 19: 38-41.

17. Kanamori H, Kanemitsu K, Miyasaka T, Ameku K, Endo S, Aoyagi T, et al. Measurement of (13)-beta-D-glucan derived from different gauze types. Tohoku J Exp Med. 2009; 217: 117-121.

18. Kimura Y, Nakao A, Tamura H, Tanaka S, Takagi H. Clinical and experimental studies of the limulus test after digestive surgery. Surg Today. 1995; 25: 790-794.

19. Mennink-Kersten MA, Ruegebrink D, Verweij PE. Pseudomonas aeruginosa as a cause of 1,3beta-D-glucan assay reactivity. Clin Infect Dis. 2008; 46: 1930-1931.

20. Marty FM, Lowry CM, Lempitski SJ, Kubiak DW, Finkelman MA, Baden LR. Reactivity of (1-->3)beta-D-glucan assay with commonly used intravenous antimicrobials. Antimicrob Agents Chemother. 2006; 50: 3450-3453.

21. Liss B, Cornely OA, Hoffmann D, Dimitriou V, Wisplinghoff H. 1,3- $\beta$-D-glucan contamination of common antimicrobials. J Antimicrob Chemother. 2016; 71: 913-915.

22. Hou TY, Wang SH, Liang SX, Jiang WX, Luo DD, Huang DH. The screening performance of serum 1,3-beta-D-glucan in patients with invasive fungal diseases: A meta-analysis of prospective cohort studies. PLoS One. 2015; 10: e0131602.

23. Koltze $A$, Rath $P$, Schöning $S$, Steinmann J, Wichelhaus TA, Bader $P$, et al. $\beta$-D-glucan screening for detection of invasive fungal disease in children undergoing allogeneic hematopoietic stem cell transplantation. J Clin Microbiol. 2015; 53: 2605-2610.

24. Lamoth F, Cruciani M, Mengoli $C$, Castagnola $E$, Lortholary $O$, Richardson $M$, et al. $\beta$-glucan antigenemia assay for the diagnosis of invasive fungal infections in patients with hematological malignancies: A systematic review and meta-analysis of cohort studies from the Third European Conference on Infections in Leukemia (ECIL-3). Clin Infect Dis. 2012; 54: 633643.

25. He S, Hang JP, Zhang L, Wang F, Zhang DC, Gong FH. A systematic review and meta-analysis of diagnostic accuracy of serum 1,3- $\beta$-D-glucan for invasive fungal infection: Focus on cutoff levels. J Microbiol Immunol Infect. 2015; 48: 351-361. 
26. Huppler AR, Fisher BT, Lehrnbecher T, Walsh TJ, Steinbach WJ. Role of molecular biomarkers in the diagnosis of invasive fungal diseases in children. J Pediatric Infect Dis Soc. 2017; 6: S32S44.

27. Lehrnbecher $T$, Robinson $\mathrm{P}$, Fisher B, Alexander S, Ammann RA, Beauchemin $M$, et al. Guideline for the management of fever and neutropenia in children with cancer and hematopoietic stem-cell transplantation recipients: 2017 update. J Clin Oncol. 2017; 35: 20822094.

28. Badiee P, Alborzi A, Karimi M, Pourabbas B, Haddadi P, Mardaneh J, et al. Diagnostic potential of nested PCR, galactomannan EIA, and beta-D-glucan for invasive aspergillosis in pediatric patients. J Infect Dev Ctries. 2012; 6: 352-357.

29. Guitard J, Tabone MD, Senghor Y, Cros C, Moissenet D, Markowicz K, et al. Detection of $\beta$-Dglucan for the diagnosis of invasive fungal infection in children with hematological malignancy. J Infect. 2016; 73: 607-615.

30. Gupta P, Ahmad A, Khare V, Kumar A, Banerjee G, Verma N, et al. Comparative evaluation of pan-fungal real-time $P C R$, galactomannan and (1-3)- $\beta$-D-glucan assay for invasive fungal infection in paediatric cancer patients. Mycoses. 2017; 60: 234-240.

31. Zhao L, Tang JY, Wang Y, Zhou YF, Chen J, Li BR, et al. Value of plasma beta-glucan in early diagnosis of invasive fungal infection in children. Zhongguo Dang Dai Er Ke Za Zhi. 2009; 11: 905-908.

32. Jaijakul S, Vazquez JA, Swanson RN, Ostrosky-Zeichner L. (1,3)- $\beta$-D-glucan as a prognostic marker of treatment response in invasive candidiasis. Clin Infect Dis. 2012; 55: 521-526.

33. Koo S, Baden LR, Marty FM. Post-diagnostic kinetics of the ( $\rightarrow 3)$ - $\beta$-D-glucan assay in invasive aspergillosis, invasive candidiasis and Pneumocystis jirovecii pneumonia. Clin Microbiol Infect. 2012; 18: E122-E127.

34. Mikulska M, Furfaro E, Del Bono V, Gualandi F, Van Lint MT, Miletich F, et al. Persistence of a positive (1,3)-beta-D-glucan test after clearance of candidemia in hematopoietic stem cell transplant recipients. Clin Vaccine Immunol. 2011; 18: 518-519.

35. Thornton CR. Detection of invasive aspergillosis. Adv Appl Microbiol. 2010; 70: 187-216.

36. Mercier T, Guldentops E, Lagrou K, Maertens J. Galactomannan, a surrogate marker for outcome in invasive aspergillosis: Finally coming of age. Front Microbiol. 2018; 9: 661.

37. Stynen D, Goris A, Sarfati J, Latgé JP. A new sensitive sandwich enzyme-linked immunosorbent assay to detect galactofuran in patients with invasive aspergillosis. J Clin Microbiol. 1995; 33: 497-500.

38. Verweij PE, Mennink-Kersten MASH. Issues with galactomannan testing. Med Mycol. 2006; 44: S179-S183.

39. Ortiz-Brizuela E, Rangel-Cordero A, Ponce-de-León A, Sierra-Madero J. False-positive results in the galactomannan Platelia ${ }^{\mathrm{TM}}$ Aspergillus assay with generic piperacillin/tazobactam. Rev Iberoam Micol. 2019; 36: 51-52.

40. Mikulska M, Furfaro $E$, Del Bono V, Raiola AM, Ratto $S$, Bacigalupo $A$, et al. Piperacillin/tazobactam $\left(\right.$ Tazocin $^{\mathrm{TM}}$ ) seems to be no longer responsible for false-positive results of the galactomannan assay. J Antimicrob Chemother. 2012; 67: 1746-1748.

41. Arboleya S, Watkins C, Stanton C, Ross RP. Gut bifidobacteria populations in human health and aging. Front Microbiol. 2016; 7: 1204. 
42. Mennink-Kersten MA, Ruegebrink D, Klont RR, Warris A, Gavini F, Op den Camp HJ, et al. Bifidobacterial lipoglycan as a new cause for false-positive platelia Aspergillus enzyme-linked immunosorbent assay reactivity. J Clin Microbiol. 2005; 43: 3925-3931.

43. Petraitiene R, Petraitis V, Witt JR, Durkin MM, Bacher JD, Wheat $L$, et al. Galactomannan antigenemia after infusion of gluconate-containing Plasma-Lyte. J Clin Microbiol. 2011; 49: 4330-4332.

44. Aceti A, Corvaglia L, Faldella G. Infant formulas thickened with carob bean gum causing falsepositive galactomannan test reactivity. Pediatr Infect Dis J. 2008; 27: 769.

45. Aubry A, Porcher R, Bottero J, Touratier S, Leblanc T, Brethon B, et al. Occurrence and kinetics of false-positive Aspergillus galactomannan test results following treatment with beta-lactam antibiotics in patients with hematological disorders. J Clin Microbiol. 2006; 44: 389-394.

46. Lehrnbecher T, Robinson PD, Fisher BT, Castagnola E, Groll AH, Steinbach WJ, et al. Galactomannan, $\beta$-D-glucan, and polymerase chain reaction-based assays for the diagnosis of invasive fungal disease in pediatric cancer and hematopoietic stem cell transplantation: A systematic review and meta-analysis. Clin Infect Dis. 2016; 63: 1340-1348.

47. Han SB, Kim SK, Lee JW, Yoon JS, Chung NG, Cho B, et al. Serum galactomannan index for early prediction of mortality in immunocompromised children with invasive pulmonary aspergillosis. BMC Infect Dis. 2015; 15: 271.

48. Kovanda LL, Desai AV, Hope WW. Prognostic value of galactomannan: Current evidence for monitoring response to antifungal therapy in patients with invasive aspergillosis. J Pharmacokinet Pharmacodyn. 2017; 44: 143-151.

49. Koo S, Bryar JM, Baden LR, Marty FM. Prognostic features of galactomannan antigenemia in galactomannan-positive invasive aspergillosis. J Clin Microbiol. 2010; 48: 1255-1260.

50. Boutboul F, Alberti C, Leblanc T, Sulahian A, Gluckman E, Derouin F, et al. Invasive aspergillosis in allogeneic stem cell transplant recipients: Increasing antigenemia is associated with progressive disease. Clin Infect Dis. 2002; 34: 939-943.

51. Miceli MH, Grazziutti ML, Woods G, Zhao W, Kocoglu MH, Barlogie B, et al. Strong correlation between serum aspergillus galactomannan index and outcome of aspergillosis in patients with hematological cancer: Clinical and research implications. Clin Infect Dis. 2008; 46: 1412-1422. 


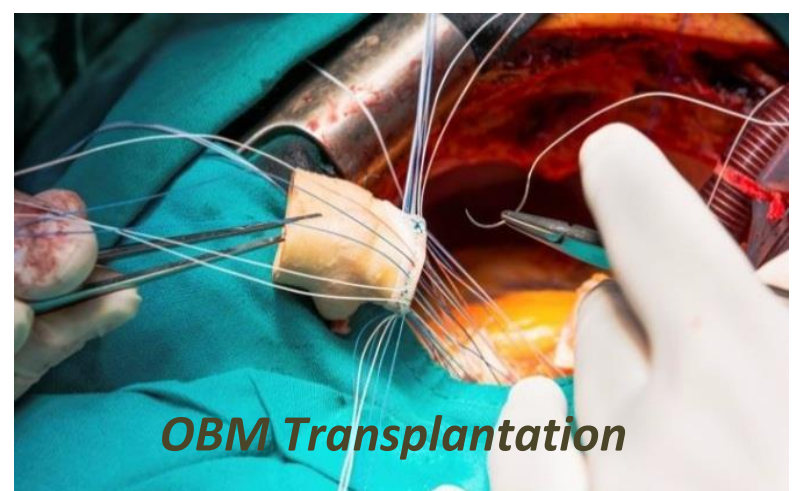

Enjoy OBM Transplantation by:

1. Submitting a manuscript

2. Joining in volunteer reviewer bank

3. Joining Editorial Board

4. Guest editing a special issue

For more details, please visit:

http://www.lidsen.com/journals/transplantation 